\title{
Aggressive Inverted Papilloma with Intracranial Invasion and Short Malignization Time
}

\author{
Peter Valentin Tomazic, M.D., ${ }^{1}$ Heinz Stammberger, M.D., ${ }^{1}$ \\ Walter Habermann, M.D., ${ }^{1}$ Christoph Schmid, M.D., ${ }^{1}$ Wolfgang Koele, M.D., ${ }^{1}$ \\ Michael Mokry, M.D., ${ }^{2}$ Verena Gellner, M.D., ${ }^{2}$ and Alfred Beham, M.D. ${ }^{3}$
}

Inverted papillomas (IP) are considered benign lesions with a prevalence up to $4 \%$ among all sinunasal tumors; however, invasive growth and varying tendency for malignization are reported in literature. We report the case of a 69-year-old woman suffering from a large, aggressively growing IP invading the orbit, skull base, and frontal lobe of the brain. Within only 3 months' time the papilloma showed transformation into an invasive carcinoma, leaving surgical therapy in vain due to explosive recurrence. Intracranial and intraorbital expansion by IP is possible despite histology not showing signs of malignancy initially. In "regular" IP close endoscopic follow-up is mandatory to not overlook recurrence harboring malignancy.
\end{abstract}

KEYWORDS: Inverted papilloma, endoscopic surgery, rapid malignization, intracranial involvement

Inverted papilloma (IP) was first described in the late 19 th century. ${ }^{1}$ It is a benign tumor characterized by inverse growth of epithelium into the nasal mucosa's stroma with an intact basal membrane separating the epithelial from the mucosal component. ${ }^{2}$ Prevalence ranges from 0.5 to $4.0 \%$ among all sinunasal tumors. ${ }^{3-5}$

IPs have a tendency for recurrence and malignant transformation. 6,7 In the past, lateral rhinotomy, Caldwell-Luc, and other external procedures were performed implicating a high comorbidity rate and dissatisfying cosmetic outcome. ${ }^{2,8,9}$ Over the past 25 years endoscopic approaches have become popular given a good visualization of the operating field with lower complication and comorbidity rates by comparably low recurrences. ${ }^{2,10}$
IPs show expansive growth throughout the nasal cavity. Transcranial or intraorbital spread is rare unless malignant transformation is present. ${ }^{2} \mathrm{We}$ report the case of an aggressive intracranially and intraorbitally invading IP with rapid transformation into focally invasive squamous cell carcinoma.

\section{CASE REPORT}

We report the case of a 69-year-old woman who presented with anosmia for 2 months as well as diplopia and exophthalmos of the right eye for 2 weeks. Recently, vertigo without spontaneous nystagmus as well as frontal cephalea had occurred. Imaging (CT and MRI) revealed a large tumor filling the entire nasal cavity including the
${ }^{1}$ Department for General Otolaryngology, Head and Neck Surgery; ${ }^{2}$ Department of Neurosurgery; ${ }^{3}$ Institute of Pathology, Medical University Graz, Graz, Austria.

Address for correspondence and reprint requests: Peter Valentin Tomazic, M.D., ENT-University Hospital Graz, Medical University Graz, Auenbruggerplatz 26/28, 8036 Graz, Austria (e-mail: peter. tomazic@medunigraz.at).
Skull Base Rep 2011;1:111-114. Copyright (C) 2011 by Thieme Medical Publishers, Inc., 333 Seventh Avenue, New York, NY 10001, USA. Tel: $+1(212)$ 584-4662.

Received: March 9, 2011. Accepted after revision: March 28, 2011. Published online: June 9, 2011.

DOI: http://dx.doi.org/10.1055/s-0031-1280738.

ISSN 2157-6971. 

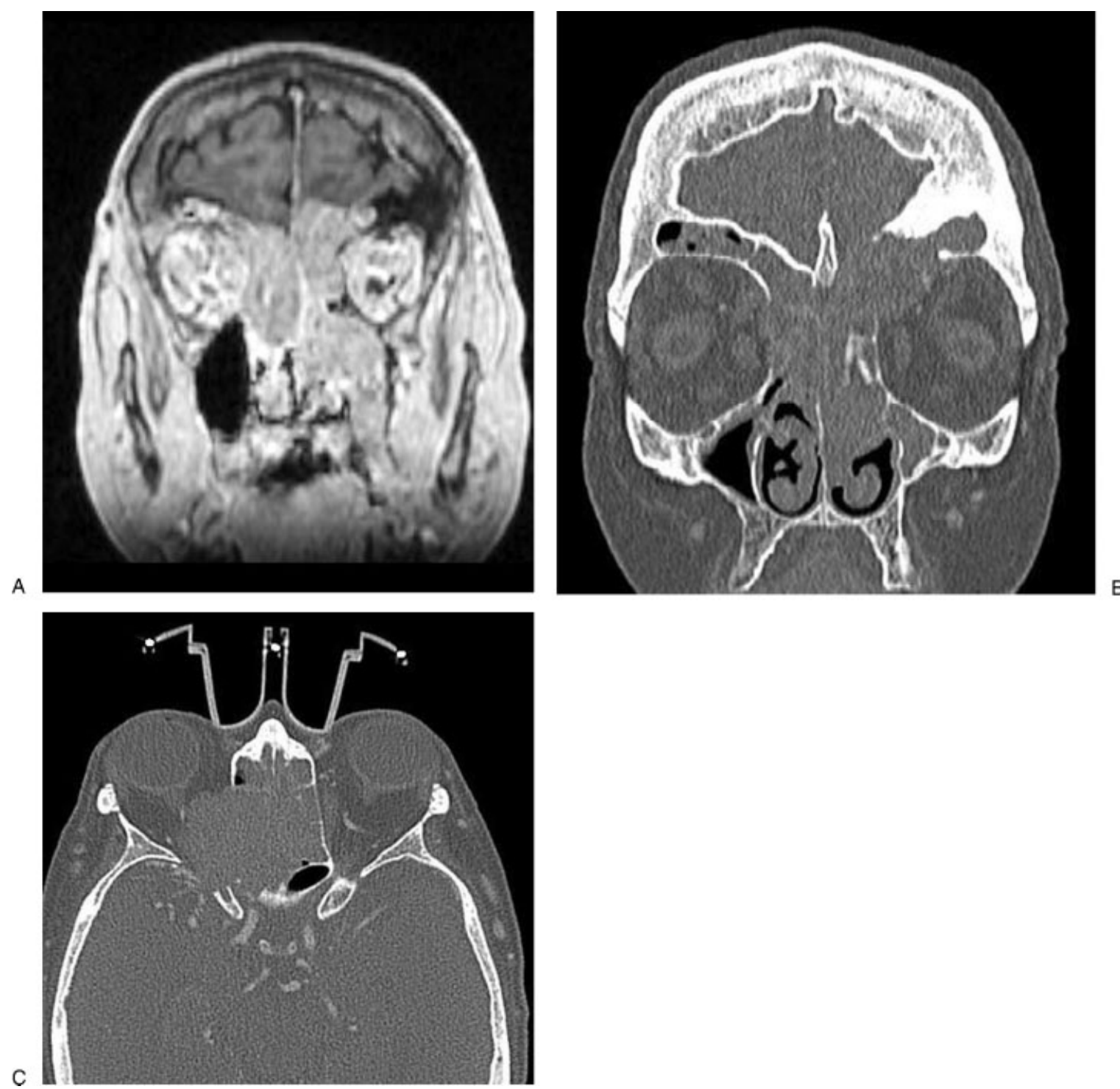

Figure 1 Preoperative $\mathrm{MRI}$ and $\mathrm{CT}$ at the time of first diagnosis. (A) Coronal preoperative MRI showing intracranial involvement of IP. (B) Coronal CT showing tumor extent into frontal sinus and intracranial invasion. (C) Axial CT showing intraorbital involvement on the right.

frontal sinus invading the right orbit. Furthermore the tumor expanded intracranially infiltrating the frontal lobe (Figs. 1A to 1C) suspicious for a sinonasal carcinoma. A biopsy resulted in IP without any signs of malignancy.

The patient was referred for surgery and further histological examinations since the preliminary histology was doubted due to the tumor's aggressive behavior.

A transnasal endoscopic approach was decided upon performing a maximal debulking including a Draf III procedure for the frontal sinuses. A consecutive craniotomy followed: Intraoperatively, the tumor eroded the right orbital roof, infiltrated the frontal lobe and both olfactory bulbs. Wide resection of tumor including infiltrated dura could be achieved with radical macro- scopic resection. Skull base reconstruction was done with fascia lata resulting in a watertight closure, which was verified endoscopically. Definite histology revealed IP with only some small foci of carcinoma in situ without invasion (Figs. 2A and 2B). In an interdisciplinary tumor conference a "watch and wait" strategy was decided upon since an effective radiation dose of 7.000 Gray would have damaged normal brain tissue to an undesired degree compared with its benefit.

Three months later a control MRI revealed explosive tumor progression in the right orbit and a re-craniotomy was performed. Intraoperatively the tumor had already invaded the fascia graft from the preceding intervention. Maximum debulking and resection of the invaded dura was done. Again, skull base was reconstructed using fascia lata. All material sent for 


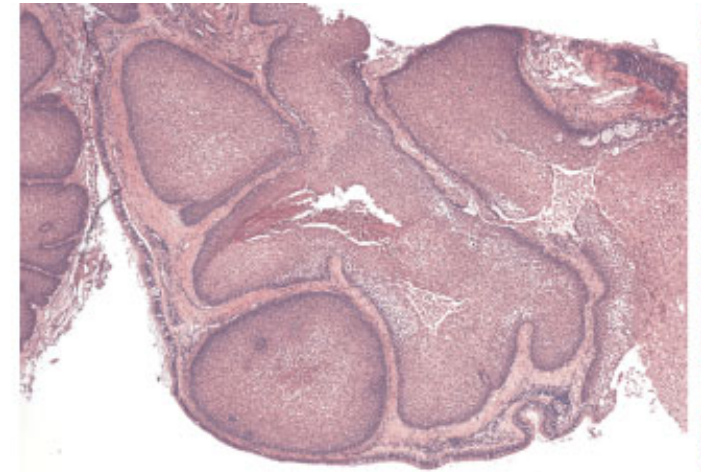

A

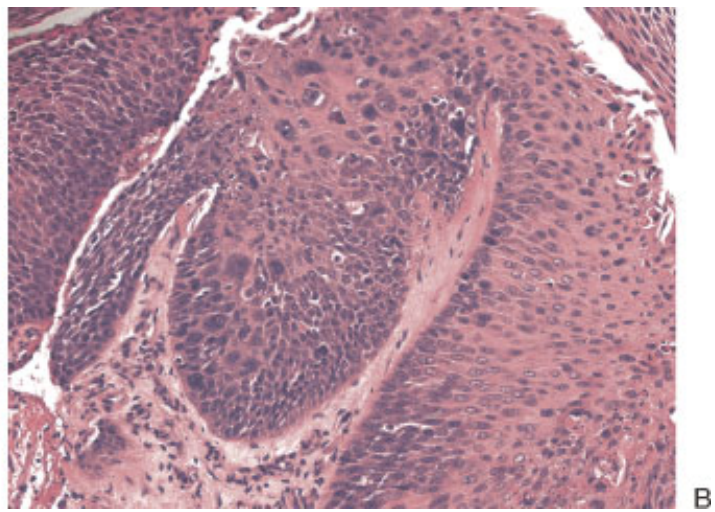

Figure 2 Histological examination after first operation. (A) Hematoxylin/eosin-stained slice under microscope showing regular inverted papilloma (IP) tissue after first operation (magnification original $\times 45$ ). (B) Hematoxylin/eosin-stained slice under microscope showing carcinoma in situ after first operation (magnification original $\times 200$ ).

histopathologic evaluation was examined meticulously to not overlook a slice containing carcinomatous tissue at an earlier stage. Definite histology revealed IP with carcinoma in situ showing one focus only of an invasive highly differentiated (G1) squamous cell carcinoma, equaling a malignization time from IP to invasive carcinoma of 3 months (Figs. 3A and 3B).

Twenty-four days after the revision craniotomy, the patient had to be revised for a subgaleal pneumatocephalus.

Because, by then, the tumor had extended beyond possible surgical and/or radiotherapeutic intervention a palliative chemotherapy was administered.

Under therapy the patient's health condition deteriorated rapidly and she died due to cerebral edema and consecutive brain stem compression only 9 months after IP had been diagnosed for the first time.

\section{DISCUSSION}

The present case should sensitize ENT doctors treating IP of its deceiving nature. As shown in our case the tumor's rapid and invasive growth pattern did not correlate with the histological findings which would have predicted a much less aggressive behavior. Although considered a benign lesion IP's growth pattern and ability to malignant transformation require extensive primary resection and meticulous follow-up., ${ }^{5,11}$ Its etiology remains unclear although association between virus infection particularly with human papilloma viruses has been proposed, whereas Llorente et $\mathrm{al}^{12}$ did not detect genetic material of neither Epstein-Barr virus (EBV) nor human papillomavirus (HPV) in their series through in situ hybridization and polymerase chain reaction.

Endoscopic surgery is the gold standard for the majority of cases providing good results with fewer complications than traditional external approaches. ${ }^{13}$ Limits of endoscopic resection depend on the localization of the tumor such as wide lateral extent in frontal sinus/orbit or intracranial involvement. ${ }^{7,11}$

IPs have a tendency for malignization after a certain period of time if untreated or left as residual tumor yielding a metachronous malignancy rate at detected recurrence of $\sim 3.6 \% .^{2}$ In our case the IP showed an aggressive growth with extensive invasion intracranially

A
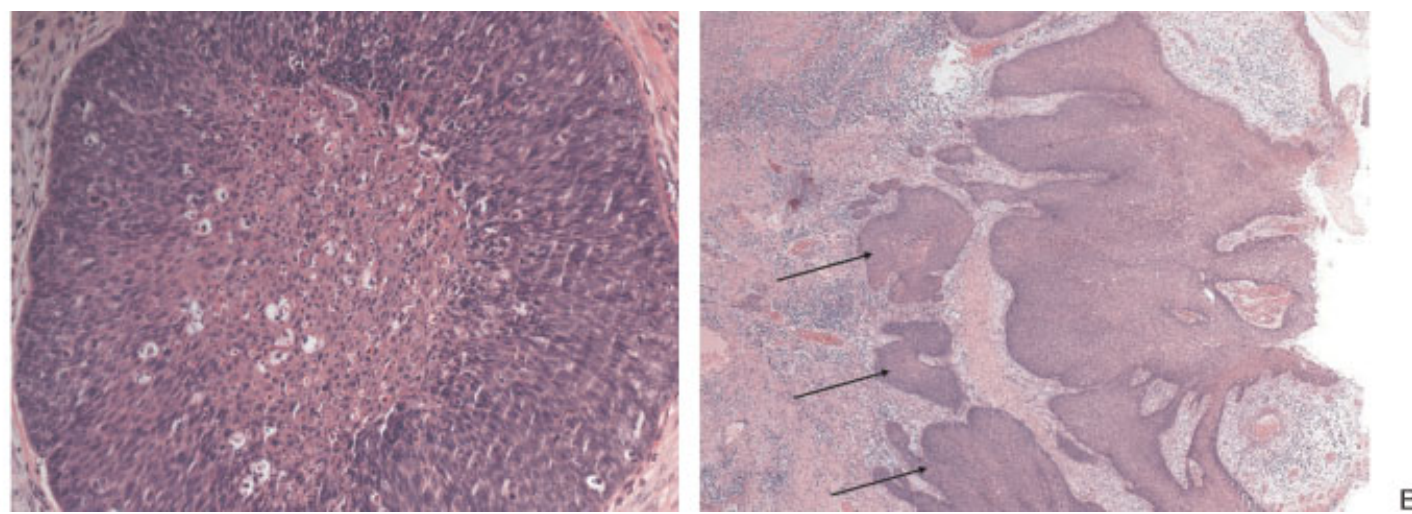

Figure 3 Histological examination 3 months after first operation. (A) Hematoxylin/eosin-stained slice under microscope showing invasive carcinoma after transcranial operation for recurrence (magnification original $\times 200$ ). (B) Hematoxylin/eosinstained slice under microscope showing invasive carcinoma with stromal invasion (marked with arrow) after transcranial operation for recurrence (magnification original $\times 45$ ). 
and intraorbitally. The time from first histological diagnosis of IP to invasive carcinoma was only 3 months which is short compared with literature. In their review, Mirza et $\mathrm{al}^{2}$ calculated a mean duration to develop into a carcinoma of 52 months (range 6 to 180 months). Nevertheless intracranial and/or orbital involvement with IPs is considered a rare condition. ${ }^{14}$ Apart from difficulty in resecting such expansive tumors, adjunctive therapy is limited in highly differentiated papillomas.

The present case impressively demonstrates how a large IP aggressively penetrated skull base and the orbit. This condition might be seen in IP which underwent broad malignant transformation, however, only one focus of our specimen revealed invasive carcinoma under the microscope with intact basal membrane in the rest of the tumor.

\section{CONCLUSION}

Although considered benign, IPs can be pitfalls because of malignant transformation, explosive growth, and intracranial and/or intraorbital invasion, despite histology does not reflect this aggressive behavior as shown in our case. Even if reported malignization rates are low with long duration, fast transformation is possible and tumor size does not reflect histological grade. Endoscopic surgery is safe and effective though limits are wide intracranial involvement, extensive (lateral) orbital invasion, and far laterally extending tumors. A close endoscopic follow-up is of utmost importance to not overlook recurrence harboring the potential of malignant transformation.

\section{REFERENCES}

1. Billroth T. Uber Den Bau Der Schleimhautpolypen. Berlin, Germany: G Reimer; 1855:11-14
2. Mirza S, Bradley PJ, Acharya A, Stacey M, Jones NS. Sinonasal inverted papillomas: recurrence, and synchronous and metachronous malignancy. J Laryngol Otol 2007;121(9): 857-864

3. Reh DD, Lane AP. The role of endoscopic sinus surgery in the management of sinonasal inverted papilloma. Curr Opin Otolaryngol Head Neck Surg 2009;17(1):6-10

4. Tomenzoli D, Castelnuovo P, Pagella F, et al. Different endoscopic surgical strategies in the management of inverted papilloma of the sinonasal tract: experience with 47 patients. Laryngoscope 2004;114(2):193-200

5. Constantino GdeT, Abdo TT, Romano FR, Voegels RL, Butugan $\mathrm{O}$. The role of endoscopic surgery in the treatment of nasal inverted papilloma. Braz J Otorhinolaryngol 2007;73(1): $65-68$

6. Sautter NB, Citardi MJ, Batra PS. Minimally invasive resection of frontal recess/sinus inverted papilloma. Am J Otolaryngol 2007;28(4):221-224

7. Holzmann D, Hegyi I, Rajan GP, Harder-Ruckstuhl M. Management of benign inverted sinonasal papilloma avoiding external approaches. J Laryngol Otol 2007;121(6):548554

8. Jameson MJ, Kountakis SE. Endoscopic management of extensive inverted papilloma. Am J Rhinol 2005;19(5):446451

9. Schlosser RJ, Mason JC, Gross CW. Aggressive endoscopic resection of inverted papilloma: an update. Otolaryngol Head Neck Surg 2001;125(1):49-53

10. Von Buchwald C, Larsen AS. Endoscopic surgery of inverted papillomas under image guidance-a prospective study of 42 consecutive cases at a Danish university clinic. Otolaryngol Head Neck Surg 2005;132(4):602-607

11. Lund VJ. Optimum management of inverted papilloma. J Laryngol Otol 2000;114(3):194-197

12. Llorente JL, Deleyiannis F, Rodrigo JP, et al. Minimally invasive treatment of the nasal inverted papilloma. Am J Rhinol 2003;17(6):335-341

13. Kamel RH. Transnasal endoscopic medial maxillectomy in inverted papilloma. Laryngoscope 1995;105(8 Pt 1):847853

14. Minovi A, Kollert M, Draf W, Bockmühl U. Inverted papilloma: feasibility of endonasal surgery and long-term results of 87 cases. Rhinology 2006;44(3):205-210 